\title{
Complement C5a inhibition improves late hemodynamic and inflammatory changes in a rat model of nonocclusive mesenteric ischemia
}

\author{
Dániel Érces, MD, PhD, ${ }^{\text {a }}$ Miklós Nógrády, MD, ${ }^{a}$ Gabriella Varga, PhD, ${ }^{a}$ Szilárd Szücs, MD, ${ }^{a}$ \\ András Tamás Mészáros, MD, ${ }^{\mathrm{a}}$ Tamás Fischer-Szatmári, MD, ${ }^{\mathrm{a}}$ Chun Cao, $\mathrm{MD},{ }^{\mathrm{a}}$ Noriko Okada, PhD, \\ Hidechika Okada, MD, PhD, ${ }^{\text {b,c }}$ Mihály Boros, MD, PhD, DSc, ${ }^{a}$ and József Kaszaki, PhD, ${ }^{\text {a }}$ Szeged \\ Hungary, and Nagoya, and Toyohashi, Japan
}

\begin{abstract}
Background. Nonocclusive mesenteric ischemia (NOMI) can evolve in a variety of low-flow states. Although the mechanisms leading to NOMI-related intestinal necrosis are largely unknown, circumstantial evidence suggests that excessive vasoconstriction and complement activation both play important roles in this process. Because targeting of the circulatory malfunction of the splanchnic area could be of therapeutic relevance, we set out to investigate the long-term effects of treatment with a complement C5 a antagonist in a rat model of partial aortic occlusion (PAO)-induced transient mesenteric hypoperfusion.

Methods. The mean arterial pressure of the splanchnic area was kept between 30 and $40 \mathrm{~mm} \mathrm{Hg}$ by 60 minutes of PAO in anesthetized male Sprague-Dawley rats. C5 a inhibitor acetyl-peptide-A (AcPepA; $4 \mathrm{mg} \mathrm{kg}^{-1}$ intravenously) or vehicle administration was initiated at the 45 th minute of PAO. After 24 hours, the animals were reanesthetized to record the macrohemodynamics and ileal microcirculation, and plasma and tissue samples were taken for determination of high-mobility group box protein-1 (HMGB-1), endothelin-1, tumor necrosis factor (TNF)- $\alpha$ levels, and small intestinal leukocyte infiltration. Epithelial structural changes were visualized by in vivo confocal laser scanning endomicroscopy.

Results. At 24 hours after PAO, mean arterial pressure, heart rate, and cardiac output were significantly greater, the intestinal intramural microcirculation was significantly impaired, and plasma HMGB-1, endothelin-1, TNF- $\alpha$ levels, the degree of epithelial damage and leukocyte infiltration was increased. The AcPepA treatment moderated the hemodynamic and microcirculatory changes, and decreased inflammatory activation and histologic signs of mucosal damage.

Conclusion. C5a inhibition ameliorated the potentially harmful local mesenteric hypoperfusion and global long-term inflammatory consequences of PAO. This approach is of promise for use in NOMIassociated situations. (Surgery 2016;159:960-71.)
\end{abstract}

From the Institute of Surgical Research, ${ }^{a}$ University of Szeged, Szeged, Hungary; the Department of Immunology, ${ }^{b}$ Nagoya City University Graduate School of Medical Sciences, Nagoya; and Choju Medical Institute, ${ }^{c}$ Fukushimura Hospital, Toyohashi, Japan

NONOCCLUSIVE MESENTERIC ISCHEMIA (NOMI) is a serious, often fatal condition that evolves in the absence of any apparent anatomic obstruction of the mesenteric circulation in a variety of low flow

Supported by research grants OTKA K75161; TÁMOP 4.2.4. A/ 2-11-1-2012-0001; NKFIH K116689.

Accepted for publication October 14, 2015.

Reprint requests: József Kaszaki PhD, Institute of Surgical Research, University of Szeged, Szokefalvi-Nagy Bela u. 6, Szeged H-6720, Hungary. E-mail: kaszaki.jozsef@med.u-szeged.hu.

$0039-6060 / \$$ - see front matter

(c) 2016 Elsevier Inc. All rights reserved.

http://dx.doi.org/10.1016/j.surg.2015.10.020 states. Although it may account for as many as $20-30 \%$ of cases of acute mesenteric ischemia, various degrees of NOMI occur in patients with unstable systemic circulatory conditions, including hemorrhagic shock, ${ }^{1}$ burn injury, ${ }^{2}$ pancreatitis, ${ }^{3}$ and the postoperative phase of cardiac and aortic surgery. ${ }^{4}$ NOMI often remains undiagnosed. ${ }^{5}$

Information on the pathogenesis of NOMI is rather scarce, but it is assumed that the potentially life-threatening structural damage of the mucosa evolves as a consequence of excessive and sustained splanchnic vasoconstriction. Nonsurgical management, however, is always challenging and precarious, because no vasodilator agent has yet 
gained widespread use for the prevention of intestinal barrier dysfunction and the subsequent development of transmural gangrene.

Conservative therapy for NOMI is nonspecific and is usually limited to hemodynamic support and the correction of biochemical alterations. Nevertheless, an increasing number of indirect data suggest a central role for the activation of complement system in the pathophysiologic mechanisms. The decrease in perfusion and oxygenation to the mucosal layer appears to activate the complement cascade, which may be one of the key elements of the innate inflammatory response, accompanied by production of the anaphylatoxins complement fragments C3a and C5a. ${ }^{6}$ Chemotaxis and activation of the neutrophil granulocytes are the main effects of C5a generation, ${ }^{7}$ whereas the release of reactive oxygen species and cytokines ${ }^{8,9}$ and smooth muscle contraction may worsen the derangements of the intramural microcirculation.

Our current knowledge of NOMI is based mostly on clinical reports or deduced from acute experimental studies involving the use of mesenteric artery occlusion. ${ }^{10}$ Our first experimental goal was, therefore, to develop a reliable in vivo rat model of NOMI of extramesenteric origin to investigate the major components of local and systemic circulatory reactions in a clinically relevant time frame. We hypothesized that the inhibition of C5a generation would offer a plausible way to influence the potentially detrimental consequences of NOMI. In an earlier study with experimental cardiac tamponade, we demonstrated activation of the complement system leading to C3 deposits in the small intestinal capillaries. ${ }^{11}$ Accordingly, we set out to characterize the effects of the C5a inhibitor peptide acetyl-peptide-A (AcPepA) ${ }^{12}$ on the macrocirculatory and microcirculatory changes and inflammatory reactions 24 hours after the initial insult leading to established low-flow conditions in the splanchnic area.

\section{MATERIALS AND METHODS}

Animals. Experiments were performed under sterile conditions on male, Sprague-Dawley rats (average weight $350 \mathrm{~g} ; n=44$ ) under sodium pentobarbital anesthesia (50 $\mathrm{mg} \mathrm{kg}^{-1}$ intraperitoneally), in adherence to the National Institutes of Health guidelines and European Union directive $2010 / 63$ for the protection of animals used for scientific purposes. The study was approved by the Ethical Committee for the Protection of Animals in Scientific Research at the University of Szeged (approval number: V/148/2013).
Pilot studies. For instrumentation, anesthetized animals were placed in a supine position on heating pads. Tracheostomy was performed to facilitate spontaneous breathing, and the right jugular vein was cannulated with PE50 tubing for drug administration and infusion of Ringer's lactate $\left(10 \mathrm{~mL} \mathrm{~kg}^{-1} \mathrm{~h}^{-1}\right)$. The left carotid artery and the left femoral artery were cannulated for mean measurements of mean arterial pressure (MAP) and heart rate (HR). A thermistor-tip catheter (PTH-01; Experimetria Ltd, Budapest, Hungary) was positioned into the ascending aorta through the right carotid artery for measurement of cardiac output (CO), using a thermodilution technique (SPEL Advanced Cardiosys 1.4, Experimetria Ltd). After a midline abdominal incision, the proximal part of the abdominal aorta was dissected free between the diaphragm and the origin of the superior mesenteric artery (SMA), and a silicone tourniquet catheter was positioned around the vessel. An ultrasonic flow-probe (1RS; Transonic Systems Inc, Ithaca, NY) was placed around the SMA to measure SMA flow. In each group, baseline macrohemodynamic parameters (MAP, CO, and SMA flow) were recorded after a 30 -minute recovery period. Hemodynamic measurements were taken at baseline, 30 minutes after the induction of partial aortic occlusion (PAO), before the release of $\mathrm{PAO}$, and at 90 and 120 minutes.

The animals were allocated randomly into 2 groups. Group $1(n=7)$ underwent 1 hour of PAO induced by controlled tightening of the tourniquet. The goal was to keep the MAP in the femoral artery continuously between 30 and $40 \mathrm{~mm} \mathrm{Hg}$. The MAP, CO, and SMA flow were recorded at baseline, 30 minutes after the induction of PAO, before the release of PAO, and at 90 and $120 \mathrm{mi}-$ nutes. Differences in MAP between the femoral $\left(\mathrm{MAP}_{\mathrm{F}}\right)$ and the carotid arteries $\left(\mathrm{MAP}_{\mathrm{C}}\right)$, ie, proximal and distal to the site of PAO, were recorded. Group $2(n=6)$ served as sham-operated controls.

Surgical preparations and experimental protocol, day 1. Animals were placed in a supine position on heating pads, and the left femoral artery was cannulated for the recording of the MAP and HR. After a midline abdominal incision, the proximal part of the abdominal aorta was dissected free between the diaphragm and the origin of the SMA, and a silicone tourniquet catheter was positioned around the vessel. An infusion of Ringer's lactate $\left(10 \mathrm{~mL} \mathrm{~kg}^{-1} \mathrm{~h}^{-1}\right)$ was administered intraperitoneally throughout the experiments. The baseline variables were determined during a 30-minute control period. Group 1 
$(n=8)$ served as the sham-operated control; PAO was induced for 1 hour by controlled tightening of the tourniquet in group $2(n=8)$ and group 3 $(n=9)$. The goal was to keep the MAP in the femoral artery continuously between 30 and $40 \mathrm{~mm} \mathrm{Hg}$. In groups 1 and 2, the vehicle for AcPepA (a 5-minute infusion of $1 \mathrm{~mL}$ saline intravenously [IV]) was administered into the tail vein, whereas in group 3, the administration of AcPePA (Biologica Co. Ltd, Nagoya, Japan) $\left(4 \mathrm{mg} \mathrm{kg}^{-1}\right.$ in $1 \mathrm{~mL}$ saline in a 5-minute infusion IV) into the tail vein started 15 minutes before the end of PAO. The animals were observed for 90 minutes; the beginning of PAO was denoted as 0 minutes. The MAP and HR were recorded 4 times (at baseline, at 30 minutes after the induction of PAO, before the release of $\mathrm{PAO}$, and 30 minutes after the end of PAO). In each group, intravital videomicroscopy was performed in the baseline condition to examine the microcirculation of the serosa of the ileum $5 \mathrm{~cm}$ proximal from the cecum (see below). After the observation period, the femoral catheter was removed carefully, the wounds were closed, and the animals were allowed to recover. In the event of any signs of hind limb ischemia, the experiments were terminated, and the animals were excluded from the study $(n=3)$.

Experimental protocol, day 2. At 24 hours after the original operation, the animals were reanesthetized (sodium pentobarbital, $50 \mathrm{mg} \mathrm{kg}^{-1}$ intraperitoneally), tracheostomy was performed to facilitate spontaneous breathing, and the right jugular vein was cannulated with PE50 tubing for drug administration and infusion of Ringer's lactate $\left(10 \mathrm{~mL} \mathrm{~kg}^{-1} \mathrm{~h}^{-1} \mathrm{IV}\right)$. The left common carotid artery was cannulated with PE50 tubing for measurements of MAP and HR. A thermistor-tip catheter (PTH-01; Experimetria Ltd) was positioned into the ascending aorta through the right common carotid artery for measurements of CO, using a thermodilution technique with a computer program (SPEL Advanced Cardiosys 1.4). The abdominal wound was reopened, and an ultrasonic flow-probe was placed around the exposed SMA (1RS; Transonic Systems Inc) to measure the SMA flow. In each group, macrohemodynamic parameters were recorded after a 30-minute recovery period, and intravital videomicroscopy was performed to examine the microcirculation of the ileal mucosa $5 \mathrm{~cm}$ proximal from the cecum. Through another incision, fluorescence confocal laser scanning endomicroscopy (CLSEM) was performed for in vivo histologic investigations. At the end of the experiments, blood samples were taken from the inferior caval vein for determination of the plasma tumor necrosis factor (TNF)- $\alpha$, high-mobility group box protein1 (HMGB-1), and endothelin-1 (ET-1) levels. Tissue samples were taken from the ileum for conventional histologic examinations and detection of leukocyte accumulation, and the animals were then humanely killed with an overdose of pentobarbital.

Hemodynamic measurements. Pressure signals (BPR-02 transducer; Experimetria Ltd) and SMA flow signals (T206 Animal Research Flowmeter; Transonic Systems Inc) were measured continuously and registered with a computerized data acquisition system (Experimetria Ltd).

Intravital videomicroscopy of the ileal microcirculation. An intravital orthogonal polarization spectral imaging technique (Cytoscan A/R, Cytometrics, Philadelphia, PA) was used for visualization of the serosal and mucosal microcirculation of the ileum. This technique uses reflected polarized light at the wavelength of the isosbestic point of oxyhemoglobin and deoxyhemoglobin $(548 \mathrm{~nm})$. Because polarization is preserved in reflection, only photons scattered from a depth of $200 \mu \mathrm{m}$ contribute to image formation. A $10 \times$ objective was placed onto the serosal surface of the ileum, and microscopic images were recorded with an S-VHS video recorder 1 (Panasonic AG-TL 700, Matsushita Electric Ind. Co. Ltd, Osaka, Japan). Quantitative assessment of the microcirculatory parameters was performed off-line by frame-toframe blinded analysis of the videotaped images. Changes in red blood cell velocity (RBCV; $\mu \mathrm{m} \mathrm{s}^{-1}$ ) in the postcapillary venules were determined in 3 separate fields by means of a computer-assisted image analysis system (IVM Pictron, Budapest, Hungary). All microcirculatory evaluations were performed by the same investigator (GV).

Plasma TNF- $\alpha$, HMGB-1, and ET-1 measurements. Blood samples $(0.5 \mathrm{~mL})$ were taken from the inferior caval vein into precooled, heparizined $\left(100 \mathrm{U} \mathrm{mL}^{-1}\right)$ polypropylene tubes, centrifuged at $1,000 \mathrm{~g}$ at $4^{\circ} \mathrm{C}$ for 30 minutes, and stored at $-70^{\circ} \mathrm{C}$ until assay. Plasma TNF- $\alpha$ concentration was determined in duplicate by means of a commercially available, enzyme-linked immunosorbent assay (Quantikine ultrasensitive ELISA kit for rat TNF$\alpha$; Biomedica Hungaria Kft, Budapest, Hungary). The plasma level of HMGB-1 was measured with a commercially available HMGB-1 ELISA kit (Shino-Test, Kanagawa, Japan). Concentrations of ET-1 in the plasma were investigated with a commercially available kit (Biochemica Hungaria Kft., Budapest, Hungary). 
Histologic investigation of tissue infiltration by leukocytes. Full-thickness ileum biopsies taken at the end of the experiments were analyzed in each group. The tissue was fixed in $6 \%$ buffered formalin, embedded in paraffin, cut into $4-\mu \mathrm{m}$ thick sections, and stained with hematoxylin and eosin. The infiltration of leukocytes was counted as the number of leukocytes in at least 20 fields of view at an original magnification of $400 \times$. Blinded evaluation was performed by the same investigator (SS).

In vivo detection of structural and microvascular damage of the mucosa-fluorescence CLSEM. The extent of microvascular damage of the terminal ileum was evaluated by means of fluorescence CLSEM (Five1; Optiscan Pty. Ltd, Melbourne, Victoria, Australia) developed for in vivo histology. Records were taken on day 2 for observation of the effects of treatment with the C5a inhibitor. The mucosal surface of the terminal ileum $5 \mathrm{~cm}$ proximal to the cecum was exposed surgically and laid flat for examination.

The microvascular structure was recorded after the IV administration of $0.3 \mathrm{~mL}$ of fluorescein isothiocyanate-dextran (FITC-dextran $150 \mathrm{kDa}$, $20 \mathrm{mg} \mathrm{mL}{ }^{-1}$ solution dissolved in saline, Sigma, St Louis, MO). The objective of the device was placed onto the mucosal surface of the ileum, and confocal imaging was performed 5 minutes after dye administration ( 1 scan per image, $1024 \times$ 1024 pixels and $475 \times 475 \mu \mathrm{m}$ per image).

The injury of the mucosal architecture was examined after topical application of the fluorescent dye acriflavin (Sigma). The surplus dye was washed off the mucosal surface of the ileum with saline 2 minutes before imaging. Nonoverlapping fields from the PAO group were compared with samples from the AcPepA-treated and control groups by using a semiquantitative scoring system relating to the following aspects:

I. The structure of the microvessels $(0=$ normal, $1=$ dye extravasation, but the vessel structure still recognizable, 2 = destruction, and the vessel structure unrecognizable);

II. Denudation of villi $(0=$ no denudation, $1=$ at least 1 area denuded per field of view, $2=$ more than 1 area denuded without a recognizable villus structure per field of view);

III. Edema $(0=$ no edema, $1=$ moderate epithelial swelling, 2 = severe edema);

IV. Shedding cells $(0=$ a normal, clear, well-defined villus structure without shedding cells, $1=$ some shedding cells, but $<30$ cells per field of view,
$2=$ more than 30 shedding cells per field of view, $3=$ severe debris);

V. Epithelial gap $(0=$ no gap, $1=$ some gaps, but $<5$ per villus, $2=$ more than 5 gaps per villus); and

VI. Longitudinal fissure on villi $(0=$ no fissure, 1 = fissure present).

Blinded analysis was performed twice separately by 2 investigators ( $\mathrm{MN}$ and $\mathrm{GV}$ ).

Statistical analysis. Data analysis was performed with a statistical software package (SigmaStat for Windows, Jandel Scientific, Erkrath, Germany). The Friedman repeated measures analysis of variance on ranks was applied within groups. Timedependent differences from the baseline for each group were assessed by Dunn's method. Differences between groups were analyzed with KruskalWallis 1-way analysis of variance on ranks, followed by Dunn's method for pairwise multiple comparison. Differences between groups in the pilot study were analyzed by the Mann-Whitney $U$ test, followed by Dunn's method for pairwise multiple comparison (Table). In the figures, median values and 75 th and 25 th percentiles are given.

\section{RESULTS}

Acute changes in hemodynamics in pilot studies. In these experiments, the MAP, CO, and the SMA flow were monitored to gain information about the circulatory changes induced by PAO and to verify the decrease in the SMA flow. During PAO, while the $\mathrm{MAP}_{\mathrm{F}}$ was between 30 and $40 \mathrm{~mm} \mathrm{Hg}$, the $\mathrm{MAP}_{\mathrm{C}}$ was initially increased significantly relative to both the baseline values of the general MAP and the sham-operated $\mathrm{MAP}_{\mathrm{C}}$ values, but it later differed significantly only from the baseline values. The CO started to decrease after 30 minutes of PAO and reached a significant difference compared with the sham-operated group and the baseline values by the end of PAO. The general MAP was increased significantly at 30 minutes after the relief of PAO and then returned gradually to the control level by the end of the observation period. The $\mathrm{CO}$ started to increase after the $\mathrm{PAO}$, and no differences were detected during the postocclusion period. The SMA flow decreased significantly during the $\mathrm{PAO}$ and remained significantly less than the baseline and sham-operated values up to the end of the experiments (Table). The AcPepA treatment did not have significant effects on the hemodynamic parameters in shamoperated animals ( $n=6$; data not shown).

Day 1, hemodynamic changes. In the shamoperated animals, there were no changes in the 
Table. Changes in mean arterial pressure $[\mathrm{mm} \mathrm{Hg}]$, cardiac output $\left(\mathrm{mL} \mathrm{min} \mathrm{mg}^{-1} \mathrm{~kg}^{-1}\right.$ and superior mesenteric artery flow $\left(\mathrm{mL} \min ^{-1}\right)$ on day 1

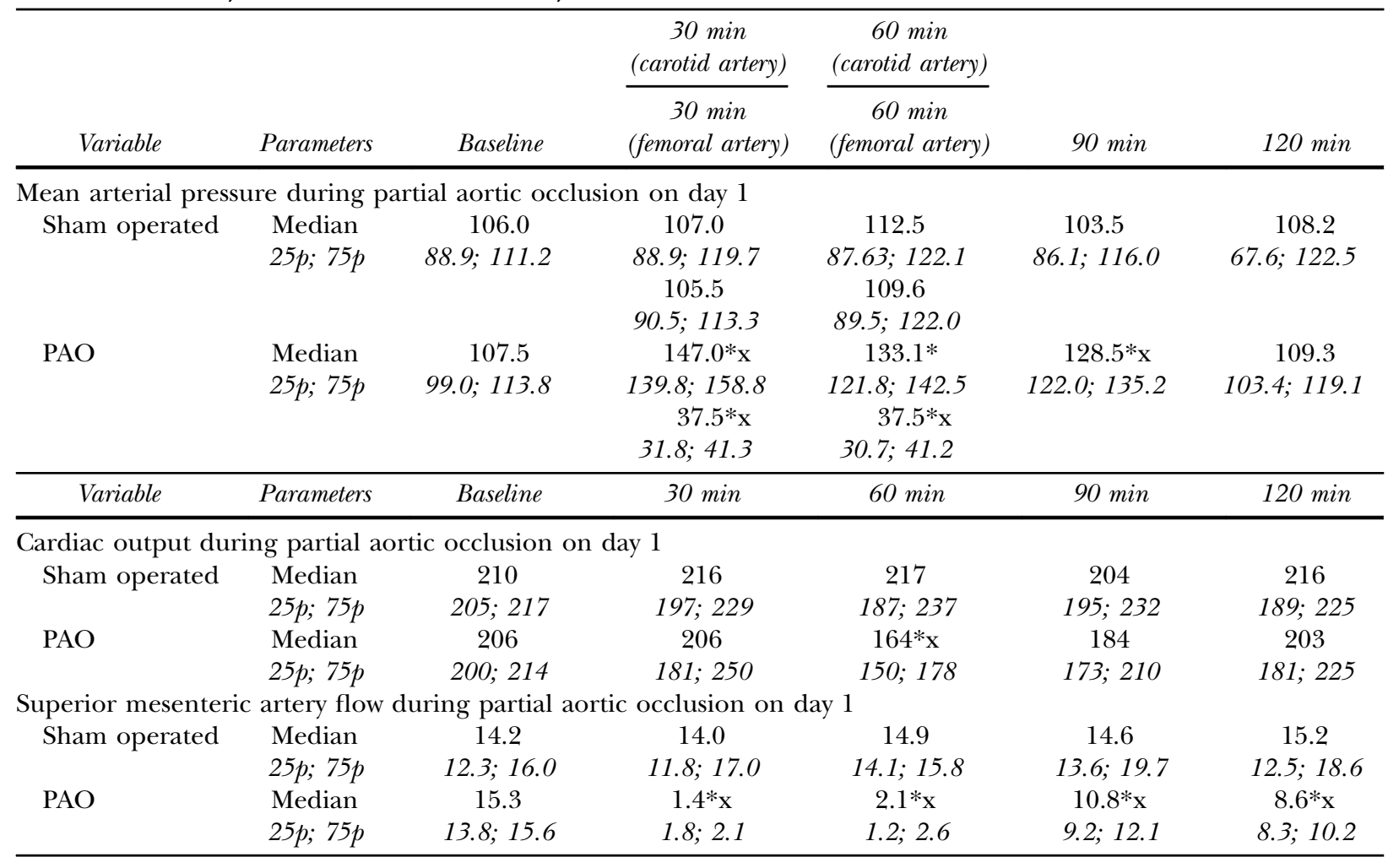

$* P<.05$ within groups vs baseline values (Friedman and Dunn test) $\mathrm{x} P<.05$ partial aortic occlusion (PAO) group vs sham-operated group (Mann-Whitney and Dunn test).

MAP on day 1 . In the groups with PAO, the MAP was kept between 30 and $40 \mathrm{~mm} \mathrm{Hg}$ for 1 hour. The MAP was significantly greater in the PAO group compared with the control group $30 \mathrm{mi}-$ nutes after the release of PAO. AcPepA did not influence these parameters. As concerns the values of the serosal microcirculation measured in the control period before PAO, there was no difference between the groups (Fig 1, A).

Day 2, hemodynamic changes. On day 2, MAP did not differ between the groups or relative to the baseline values measured on day 1 . The operative interventions caused an increase in HR by day 2 . Nonetheless, the HR was significantly greater in the PAO group compared with the sham-operated animals, and the HR did not increase after AcPepA relative to that for the sham-operated group. The SMA flow in the treated group remained at the control level, whereas in the nontreated group it was significantly greater in comparison with the sham-operated group. The increased CO was significantly decreased by C5a inhibitor on day 2 (Figs 1 and 2). On day 2, both the serosal and mucosal RBCV were significantly decreased in comparison with the sham-operated group. AcPepA resulted in a significantly greater $\mathrm{RBCV}$ (Fig 3, $A$ and $B$ ).

Changes in biochemical parameters. Concentrations of the inflammatory mediators TNF- $\alpha$ and HMGB-1 were significantly increased 24 hours after the ischemic insult. The C5a inhibitor treatment decreased the levels of both TNF- $\alpha$ and HMGB-1 (Fig 4, $A$ and $B$ ). The level of ET-1, an important vasoconstrictor that can be responsible for the impairment of the mucosal microcirculation, was also increased on the day after PAO (Fig 3, C). The administration of AcPepA decreased the ET-1 concentration significantly.

Tissue leukocyte accumulation. On day 2 of the experiments, a significant leukocyte accumulation was observed in the PAO group compared with the sham-operated animals. The administration of AcPepA significantly decreased the leukocyte infiltration of the small intestinal wall (Fig 4, C).

Microvascular and structural damage of the mucosa. The microvessels in the villi were visualized by FITC-dextran administration, and the 


\section{A}
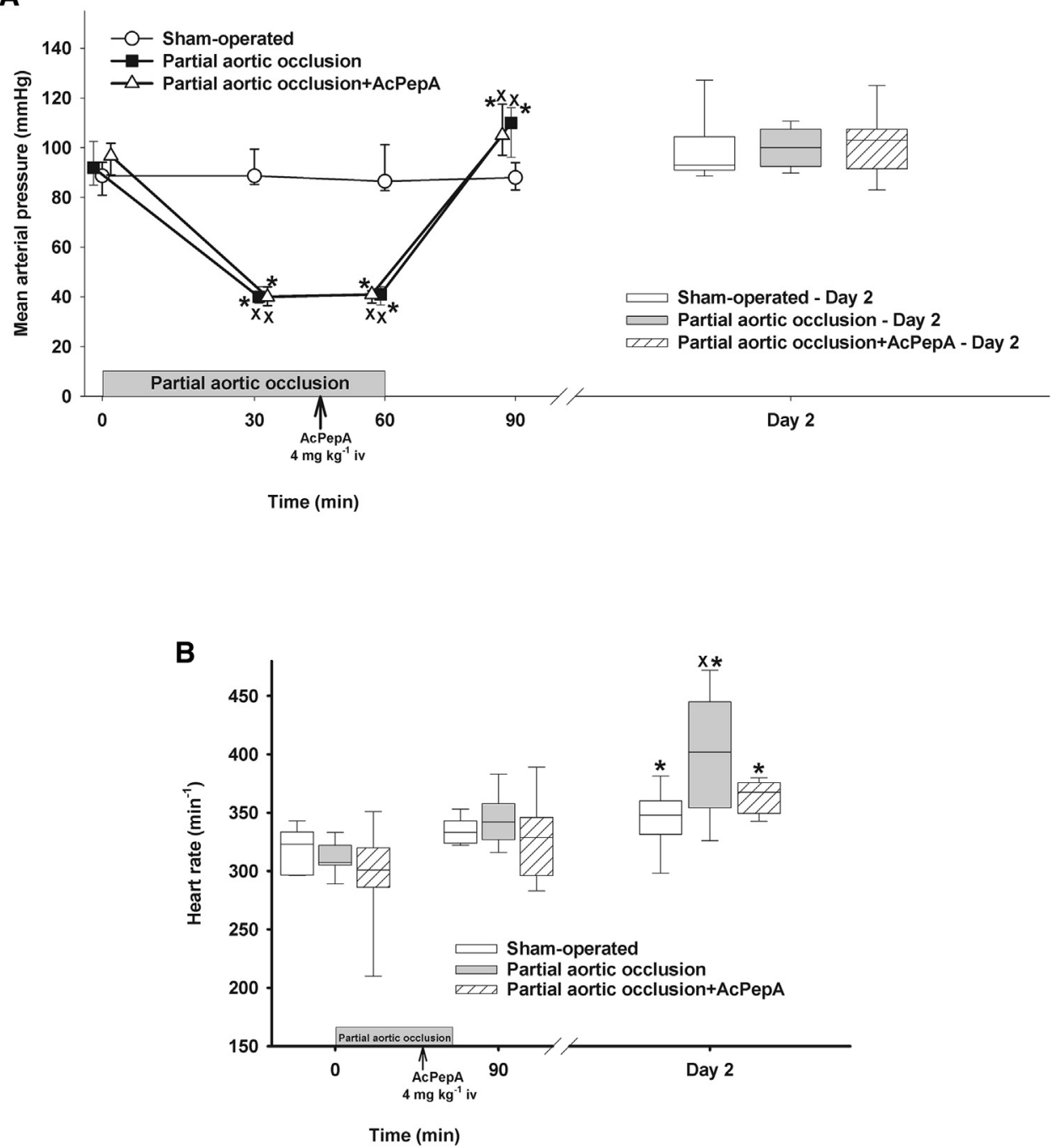

Fig 1. Changes in mean arterial pressure in the sham-operated (empty circles joined by a thin continuous line; empty box), partial aortic occlusion (PAO; black squares joined by a continuous line; gray box), and PAO+acetyl-peptide-A (PAO+AcPepA; empty triangles with continuous line; striped empty box) groups $(A)$. Changes in heart rate in the sham-operated (empty box), PAO (gray box) and PAO+AcPepA (striped empty box) groups $(B)$. The plots demonstrate the median (horizontal line in the box) and the 25 th and 75 th percentiles. $* P<.05$ for groups versus baseline values, ${ }^{\mathrm{x}} P<.05$ for groups versus sham-operated group values.

morphology of the mucosa was examined with acriflavin staining. In the PAO group, the CLSEM evaluation demonstrated significant tissue damage in contrast with the sham-operated group (Fig 5). The vessel structure was disorganized, and fluorescent dye leakage could be observed. The acriflavin staining of the surface of the villi revealed the presence of longitudinal fissures and epithelial gaps and a large amount of debris (Fig 6, $A-C$ ).

Administration of the C5a inhibitor AcPepA decreased the mucosal damage and significantly influenced the changes in both the microvascular structure and the epithelial morphology of the small intestine. These changes were more emphasized than in the sham-operated group, but the degree of injury was decreased, as shown by the decreased level of dye leakage relative to the nontreated PAO group (Fig 6, $D-F$ ).

\section{DISCUSSION}

NOMI is an often nonappreciated complication of low-flow conditions ${ }^{5}$ and a better understanding of the process is demanded if the current therapeutic possibilities are to be improved. The methods used at present to treat NOMI, such as the direct application of vasodilator agents into 


\section{A}

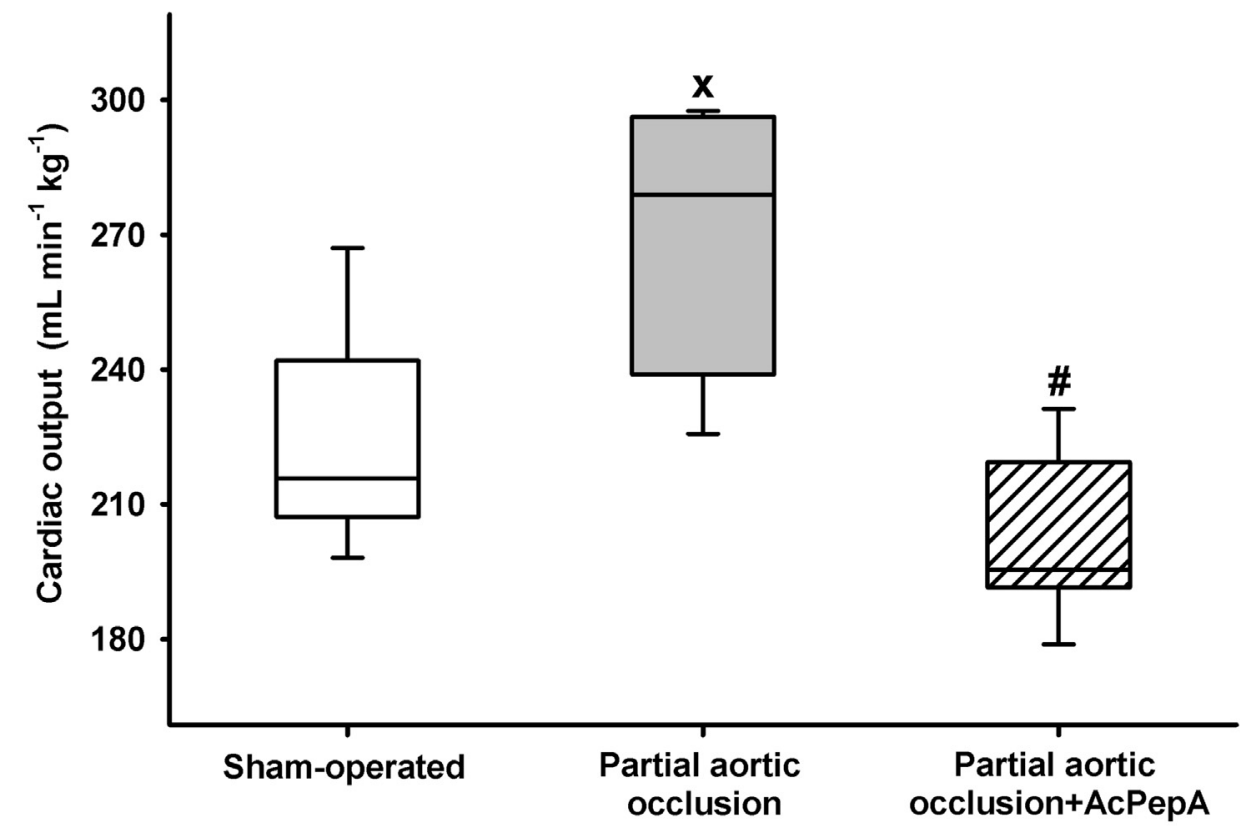

B

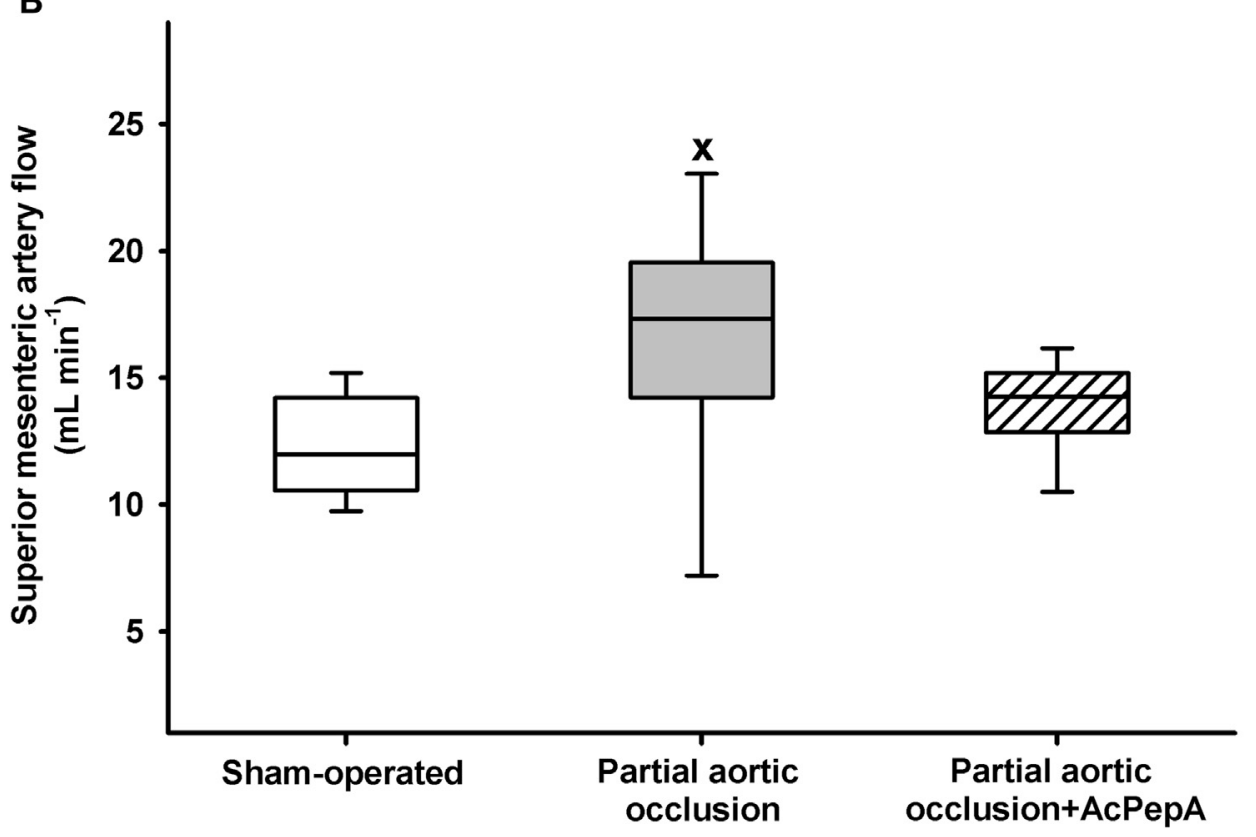

Fig 2. Changes in cardiac output index $(A)$ and in SMA flow $(B)$ in the sham-operated (empty box), partial aortic occlusion (PAO; gray box), and PAO+acetyl-peptide-A (PAO+AcPepA; striped empty box) groups. The plots demonstrate the median (horizontal line in the box) and the 25 th and 75 th percentiles. ${ }^{\mathrm{x}} P<.05$ for groups versus sham-operated group values. ${ }^{\#} P<.05$ between AcPepA-treated group versus PAO group.

the SMA, involve highly technical requirements, ${ }^{13}$ which may limit the relevance of otherwise effective vasoactive treatment.
This study involved the first application of a rat model, with which the main clinical features of NOMI could be reproduced. The key elements of 
A

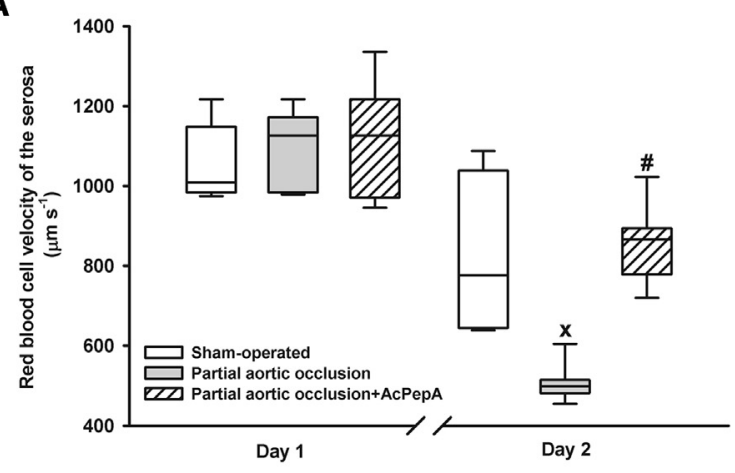

B

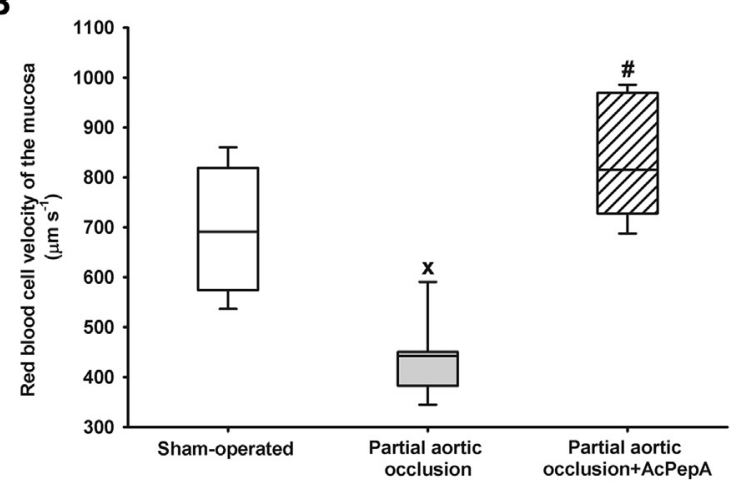

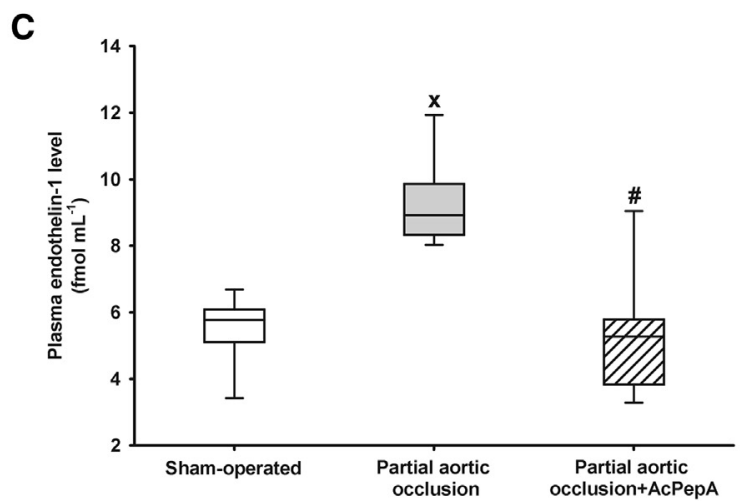

Fig 3. Changes in serosal $(A)$, mucosal $(B)$, RBCV and plasma ET-1 level $(C)$ in the sham-operated (empty box), partial aortic occlusion (PAO; gray box), and PAO+acetyl-peptide-A (PAO+AcPepA; striped empty box) groups. The plots demonstrate the median (horizontal line in the box) and the 25th and 75 th percentiles. ${ }^{\mathrm{x}} P<.05$ for groups versus shamoperated group values. ${ }^{\#} P<.05$ between AcPepA-treated group versus PAO group.

this model were a persistently decreased SMA flow after an extramesenteric insult and a low level of invasiveness, which makes long-term observations possible. In this context, we obtained conclusive evidence of a significantly decreased SMA blood flow after partial occlusion of the subdiaphragmatic aorta, which persisted during the 24-hour postocclusion period, despite the compensated CO and MAP (Table). It should be added that the late hemodynamic changes were characteristic of a systemic inflammatory response syndrome. Although the MAP was in the normal range, the $\mathrm{HR}$ and $\mathrm{CO}$ were significantly increased as a part of the compensatory mechanism. During the early phase of systemic inflammation, the oxygen demand was in all likelihood enhanced owing to the high metabolic rate. To meet this need, oxygen delivery was increased, mostly through an increase in CO. Nonetheless, we suggest that the oxygenation of the cells cannot be improved without a properly functioning microcirculation, and a deterioration of the capillary perfusion will prevent the oxygen consumption despite the seemingly increased delivery. In our model, the small intestinal microcirculation was significantly impaired 24 hours after the insult, as shown by the decreased RBCV at both the serosal and mucosal surfaces. This observation suggests that microcirculatory damage was present in the gastrointestinal wall despite the normal or increased SMA flow. ${ }^{14}$

Activation of the complement system is a key element for the induction of ischemia-reperfusion injury. During the process, complement activation can take place by all 3 known routes (classic, lectin, and alternative). Activation of the complement cascade system is a complex process that results in the formation of the membrane attack complex, an important component of the innate immune system. ${ }^{6}$

During the process, however, biologically active toxic molecules are produced such as the anaphylatoxins C3a and C5a. One of the most important direct effects of C5a, a potent chemoattractant, is the accumulation of neutrophilic leukocytes. 
A

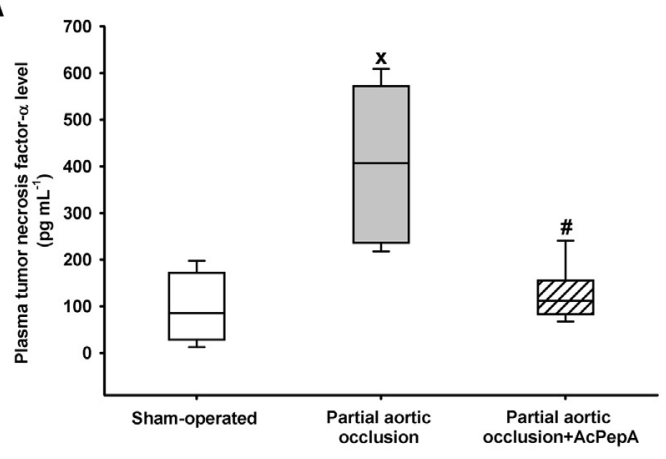

B

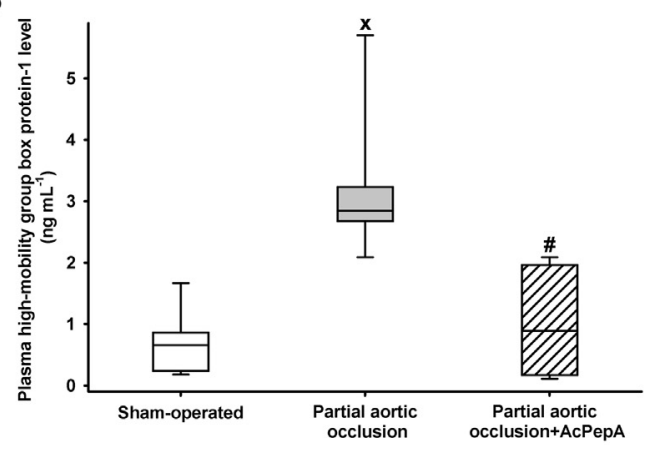

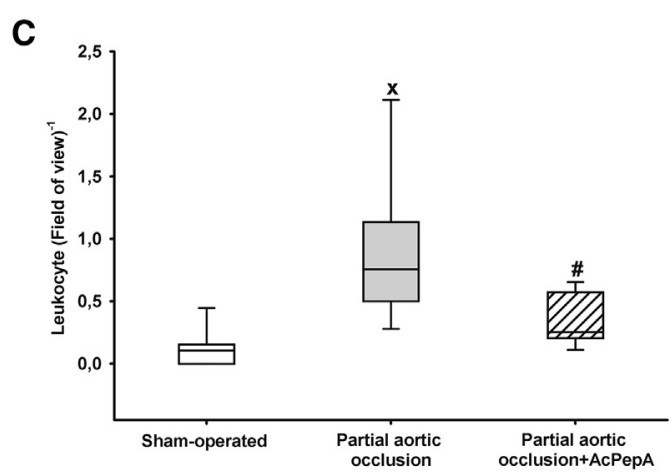

Fig 4. Changes in plasma tumor necrosis factor- $\alpha(A)$, high-mobility group box protein-1 $(B)$ levels, and small intestinal leukocyte infiltration $(C)$ in the sham operated (empty box), partial aortic occlusion (PAO; gray box) and PAO+acetylpeptide-A (PAO+AcPepA; striped empty box) groups. The plots demonstrate the median (horizontal line in the box) and the 25 th and 75 th percentiles. ${ }^{\mathrm{x}} P<.05$ for groups versus sham-operated group values; ${ }^{\#} P<.05$ between AcPepA-treated group versus PAO group.

Activated neutrophils can contribute to the increased level of tissue injury by releasing reactive oxygen species and proinflammatory agents. ${ }^{6}$ This process is also obvious in our experimental setup; in addition to the presence of increased neutrophil infiltration as a consequence of the PAO, the occurrence of tissue damage is verified by the CLSEM records. The C5a inhibitor that we used decreased these changes, similarly to known C5a receptor antagonists and $\mathrm{C} 5 \mathrm{a}$ antibodies.

The considerable number of studies on C5a receptor agonists and $\mathrm{C} 5 \mathrm{a}$ antibodies illustrate the great demand for effective C5a antagonists. ${ }^{6,15,16}$ Despite the promising experimental results, very few of these agents have found their way into clinical applications, and they have very limited efficacy. The discrepancy between the experimental and clinical results may be owing to the large amount of C5a receptors resulting from upregulation during inflammatory processes. Despite treatment with C5a receptor agonists, there are still sufficient C5aRs to maintain function. ${ }^{12}$ As concerns the C5a antibodies, the possible cross- reactions with other complement components may reduce the efficacy of the treatment by scavenging the C5a antibodies or causing undesirable side effects.

The C5a inhibitor AcPepA, however, was designed to cover the receptor-binding region of the human C5a, and its high-affinity binding was proved in vitro. Moreover, AcPepA is effective against C5a generation in several species, including rodents, swine, and primates, ${ }^{12,17-19}$ and decreases the hypersensitivity reaction in human lung tissues in vitro. ${ }^{20}$ The wide variety of species in which AcPepA has proved effective suggests its promise as regards the issue of the translation of experimental and clinical results.

In the AcPepA-treated group, the small intestinal RBCV was significantly greater on day 2 , and the improved microcirculation was present without increases in CO and SMA flow, which may reflect a better efficacy of oxygen extraction. The decreased ET-1 level after AcPepA treatment may have contributed to the improvement of the small intestinal microcirculation, and the altered proinflammatory 


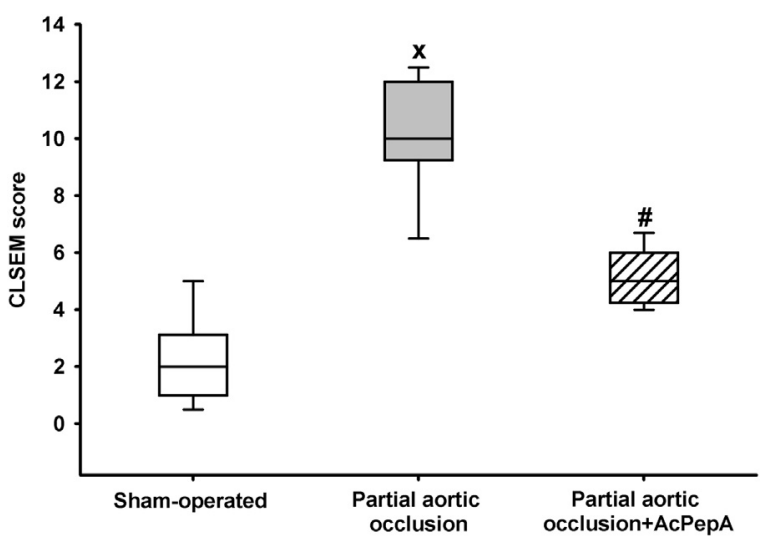

Fig 5. Level of mucosal injury according to the confocal laser scanning endomicroscopy (CLSEM) in vivo histologic records in the sham-operated (empty box), partial aortic occlusion (PAO; gray box), and PAO+acetylpeptide-A (PAO+AcPepA; striped empty box) groups. The plots demonstrate the median (horizontal line in the box) and the 25th and 75th percentiles. ${ }^{\mathrm{x}} P<.05$ for groups versus sham-operated group values; ${ }^{\#} P<.05$ between AcPepA-treated group and PAO group.

cytokine profile may also be indicative of a decrease in inflammatory activation as a consequence of C5a inhibitor therapy. ET-1 is among the most important vasoconstrictive factors in the splanchnic microcirculation, and administration of a selective endothelin type A receptor antagonist improves the small intestinal microcirculation significantly under low-flow conditions. ${ }^{21}$ More important, the excessive release of ET-1 enhances leukocyte activation in the postcapillary venules of the small intestine, ${ }^{22}$ which may exacerbate the local tissue damage further.

The plasma levels of HMGB-1 and TNF- $\alpha$ are another important feature of our model, and these findings are in accord with the clinical observations. ${ }^{23}$ A high level of TNF- $\alpha$ can impair directly gastrointestinal epithelial tight junctions, ${ }^{24}$ and a decreased level of TNF- $\alpha$ in the AcPepA-treated group may, therefore, not only be a direct sign of the alleviated inflammatory cascade activation, but may also be associated indirectly with the preservation of the barrier function. Similarly, increased output of HMGB-1 can amplify the inflammatory signals, and a significant decrease in the plasma level of HMGB-1 may be a key sign of the decreased systemic inflammatory response. ${ }^{21}$ HMGB-1 is a highly potent alarmin originating from necrotic cells or actively released from immune cells, which causes leukocyte recruitment and the release of other inflammatory cytokines. ${ }^{25}$ The release is mediated directly by C5a through
C5L2 receptors, and the blockade or absence of C5L2 receptors improves the survival in murine sepsis. ${ }^{26}$ The net result of a decrease in the release of HMGB-1 and TNF- $\alpha$ is decreased leukocyte accumulation. This was verified by traditional histology; the level of leukocyte infiltration was significantly less in the small intestinal wall in the AcPepA-treated group. All these macrocirculatory and microcirculatory and inflammatory effects of PAO culminated in visible damage of the mucosa (Fig 5), whereas C5a inhibition resulted in a significant decrease in tissue damage with a preserved villus structure and microvascular architecture, demonstrated by in vivo histology.

In general, it must be acknowledged that rat models have a number of limitations from the aspect of the extrapolation of experimental animal data to the human patient, such as differences in age, comorbidities, the timing of treatments, dosages and dose-dependent efficacies, and the lack of more complex intensive care in the animals. ${ }^{27}$ It is also important to note certain additional limitations of the present experimental model. One of these is the significantly increased $\mathrm{MAP}_{\mathrm{C}}$ that develops proximal to the site of the PAO. This increase in $\mathrm{MAP}_{\mathrm{C}}$ approximates the upper limits of the cerebral autoregulation ${ }^{28}$ for a short period, during the first 30 minutes of the PAO. Nevertheless, this rat model is tolerable, because no animals died during the first 24 hours, and none showed any signs of central nervous system injury. Another problematic point is the need for invasive measurement of blood pressure in the femoral artery because this provides more precise data for the adjustment of PAO compared with noninvasive measurements. During the design of the model, we took into consideration the welldeveloped collateral vascularization in the rat hind limb, which can provide adequate tissue perfusion even after ligation of the femoral artery. ${ }^{29,30}$ Nevertheless, before the recovery period, the animals were kept under observation and, in the event of any signs of permanent hind limb ischemia, such as cyanosis or stiffness, the experiment was terminated, and the animal was excluded from the study. The low number of such animals $(n=3)$ suggests that ligation of the femoral artery is possible in these studies.

In conclusion, we characterized some of the potentially detrimental circulatory and proinflammatory consequences of nonocclusive mesenteric hypoperfusion. In this experimental setup, a single IV dose of the C5a antagonist AcPepA improved the local circulatory changes and decreased the secondary mucosal damage in a 

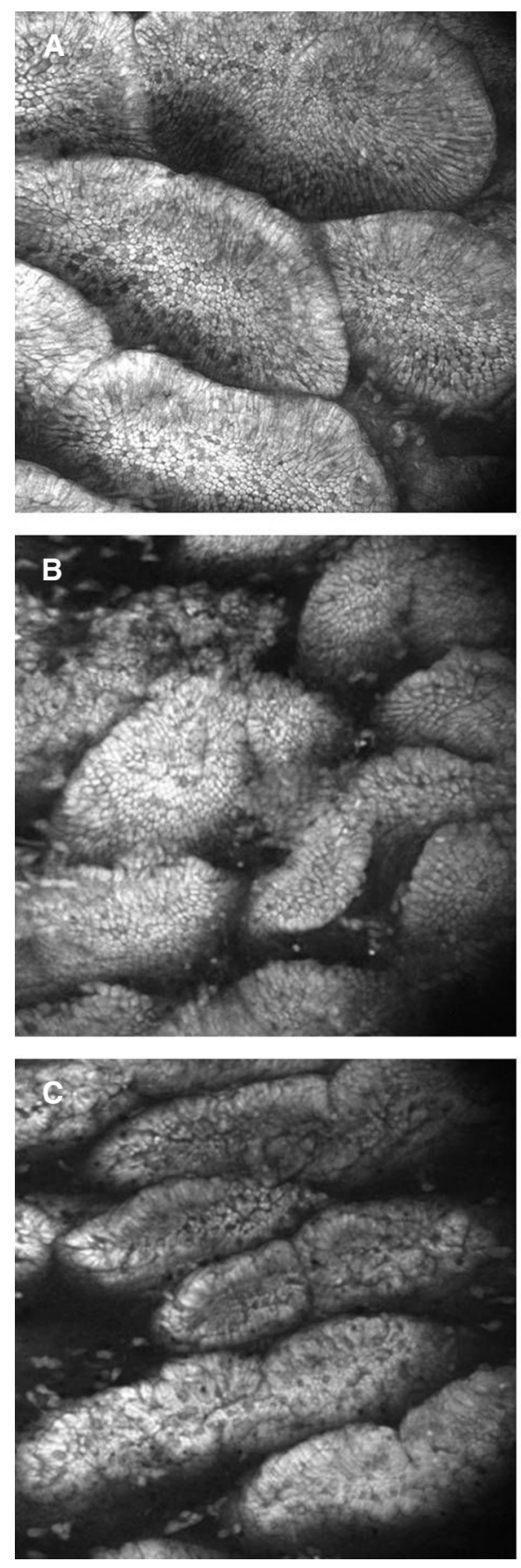
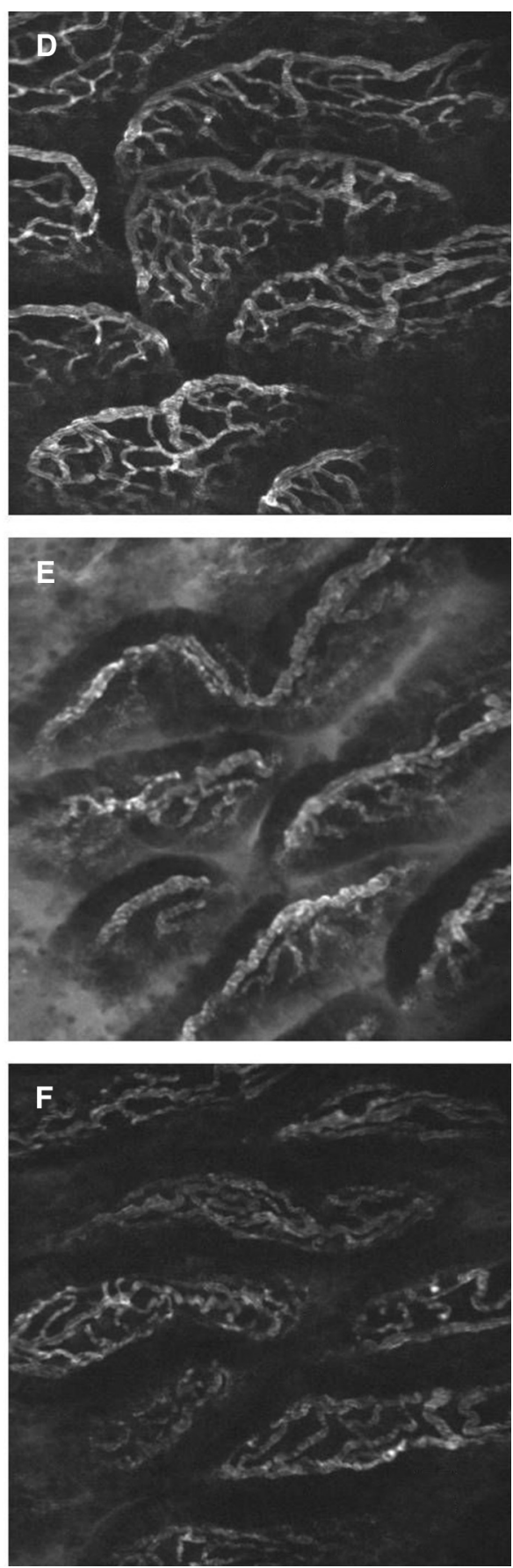

Fig 6. In vivo histologic (confocal laser scanning endomicroscopy; CLSEM) records showing the changes in the epithelial surface 24 hours after the partial aortic occlusion (PAO; acriflavin staining) in the sham-operated $(A)$, PAO $(B)$, and $\mathrm{PAO}+$ acetyl-peptide-A (PAO+AcPepA; $C$ ) groups. Changes in the microvascular structure were visualized with CLSEM after intravenous administration of fluorescein isothiocynate (FITC)-dextran in the sham operated $(D)$, PAO $(E)$, and PAO+AcPepA $(F)$ groups $(475 \times 475 \mu \mathrm{m}$ per image $)$.

relatively wide time frame, at least 24 hours after the insult. The relevance of these data in the clinical situation in man remains unknown, but this approach is of promise for use in NOMIassociated situations.
D.E. and M.N. contributed equally to this work. The authors are grateful to Ms Ágnes Fekete, Csilla Mester, Nikolett Beretka, and Lilla Kovács for skillful assistance. This research was supported by the European Union and the State of Hungary, co-financed by the European 
Social Fund in the framework of TÁMOP 4.2.4. A/2-11-12012-0001'National Excellence Program'. The study was also supported by Hungarian Science Research Fund OTKA K75161 and National Research, Development and Innovation Office-NKFIH K116689. Competing interests: none declared.

\section{REFERENCES}

1. Toung T, Reilly PM, Fuh KC, Ferris R, Bulkley GB. Mesenteric vasoconstriction in response to hemorrhagic shock. Shock Augusta Ga 2000;13:267-73.

2. Muschitz GK, Fochtmann A, Keck M, Ihra GC, Mittlböck M, Lang S, et al. Non-occlusive mesenteric ischaemia: the prevalent cause of gastrointestinal infarction in patients with severe burn injuries. Injury 2015;46:124-30.

3. Hirota M, Inoue K, Kimura Y, Mizumoto T, Kuwata K, Ohmuraya M, et al. Non-occlusive mesenteric ischemia and its associated intestinal gangrene in acute pancreatitis. Pancreatol Off J Int Assoc Pancreatol IAP Al 2003;3: 316-22.

4. Klotz S, Vestring T, Rötker J, Schmidt C, Scheld HH, Schmid C. Diagnosis and treatment of nonocclusive mesenteric ischemia after open heart surgery. Ann Thorac Surg 2001;72:1583-6.

5. Trompeter M, Brazda T, Remy CT, Vestring T, Reimer P. Non-occlusive mesenteric ischemia: etiology, diagnosis, and interventional therapy. Eur Radiol 2002;12:1179-87.

6. Guo RF, Ward PA. Role of C5a in Inflammatory Responses. Annu Rev Immunol 2005;23:821-52.

7. Ehrengruber MU, Geiser T, Deranleau DA. Activation of human neutrophils by C3a and C5A Comparison of the effects on shape changes, chemotaxis, secretion, and respiratory burst. FEBS Lett 1994;346:181-4.

8. Sacks T, Moldow CF, Craddock PR, Bowers TK, Jacob HS. Oxygen radicals mediate endothelial cell damage by complement-stimulated granulocytes. An in vitro model of immune vascular damage. J Clin Invest 1978; 61:1161-7.

9. Ember JA, Sanderson SD, Hugli TE, Morgan EL. Induction of interleukin-8 synthesis from monocytes by human C5a anaphylatoxin. Am J Pathol 1994;144:393-403.

10. Gonzalez LM, Moeser AJ, Blikslager AT. Animal models of ischemia-reperfusion-induced intestinal injury: progress and promise for translational research. Am J Physiol - Gastrointest Liver Physiol 2015;308:G63-75.

11. Vass A, Süveges G, Érces D, Nógrády M, Varga G, Földesi I, et al. Inflammatory activation after experimental cardiac tamponade. Eur Surg Res Eur Chir Forsch Rech Chir Eur 2013;51:1-13.

12. Okada N, Asai S, Hotta A, Miura N, Ohno N, Farkas I, et al. Increased Inhibitory Capacity of an Anti-C5a Complementary Peptide Following Acetylation of N-terminal Alanine. Microbiol Immunol 2007;51:439-43.

13. Weiss G, Lippert H, Meyer F. Successful Management of Non-Occlusive Mesenteric Ischemia (Nomi) - Case Report. Pol J Surg 2012;84:214-8.

14. Tao W, Zwischenberger JB, Nguyen TT, Vertrees RA, McDaniel LB, Nutt LK, et al. Gut mucosal ischemia during normothermic cardiopulmonary bypass results from blood flow redistribution and increased oxygen demand. J Thorac Cardiovasc Surg 1995;110:819-28.

15. Amsterdam EA, Stahl GL, Pan HL, Rendig SV, Fletcher MP, Longhurst JC. Limitation of reperfusion injury by a monoclonal antibody to C5a during myocardial infarction in pigs. Am J Physiol - Heart Circ Physiol 1995;268:H448-57.

16. Harkin DW, Romaschin A, Taylor SM, Rubin BB, Lindsay TF. Complement c5a receptor antagonist attenuates multiple organ injury in a model of ruptured abdominal aortic aneurysm. J Vasc Surg 2004;39:196-206.

17. Fujita E, Farkas I, Campbell W, Baranyi L, Okada H, Okada N. Inactivation of C5a anaphylatoxin by a peptide that is complementary to a region of C5a. J Immunol 2004;172:6382-7.

18. Érces D, Nógrády M, Nagy E, Varga G, Vass A, Süveges G, et al. Complement C5A antagonist treatment improves the acute circulatory and inflammatory consequences of experimental cardiac tamponade. Crit Care Med 2013;41: e344-51.

19. Okada H, Imai M, Ono F, Okada A, Tada T, Mizue Y, et al. Novel Complementary Peptides to Target Molecules. Anticancer Res 2011;31:2511-6.

20. Abe M, Hama H, Shirakusa T, Iwasaki A, Ono N, Kimura N, et al. Contribution of anaphylatoxins to allergic inflammation in human lungs. Microbiol Immunol 2005;49:981-6.

21. Szabó A, Suki B, Csonka E, Eszlári E, Kucsa K, Vajda K, et al. Flow motion in the intestinal villi during hemorrhagic shock: a new method to characterize the microcirculatory changes. Shock Augusta Ga 2004;21:320-8.

22. Boros M, Massberg S, Baranyi L, Okada H, Messmer K. Endothelin 1 induces leukocyte adhesion in submucosal venules of the rat small intestine. Gastroenterology 1998; 114:103-14.

23. Cremer J, Martin M, Redl H, Bahrami S, Abraham C, Graeter T, et al. Systemic inflammatory response syndrome after cardiac operations. Ann Thorac Surg 1996; 61:1714-20.

24. Shen L, Turner JR. Role of Epithelial Cells in Initiation and Propagation of Intestinal Inflammation. Eliminating the static: tight junction dynamics exposed. Am J Physiol - Gastrointest Liver Physiol 2006;290:G577-82.

25. Ulloa L, Messmer D. High-mobility group box 1 (HMGB1) protein: friend and foe. Cytokine Growth Factor Rev 2006; 17:189-201.

26. Rittirsch D, Flierl MA, Nadeau BA, Day DE, Huber-Lang M, Mackay CR, et al. Functional roles for C5a receptors in sepsis. Nat Med 2008;14:551-7.

27. Esmon CT. Why do animal models (sometimes) fail to mimic human sepsis? Crit Care Med 2004;32(5 Suppl): S219-22.

28. Paulson OB, Strandgaard S, Edvinsson L. Cerebral autoregulation. Cerebrovasc Brain Metab Rev 1990;2:161-92.

29. Brevetti LS, Paek R, Brady SE, Hoffman JIE, Sarkar R, Messina LM. Exercise-Induced Hyperemia Unmasks Regional Blood Flow Deficit in Experimental Hindlimb Ischemia. J Surg Res 2001;98:21-6.

30. Hellingman AA, Bastiaansen AJ, de Vries MR, Seghers L, Lijkwan MA, Löwik CW, et al. Variations in Surgical Procedures for Hind Limb Ischaemia Mouse Models Result in differences in Collateral Formation. Eur J Vasc Endovasc Surg 2010;40:796-803. 